\title{
Obliged Removal of the Percutaneous Fixation System on the Thoracolumbar Junction in Patients with Idiopathic Scoliosis
}

\author{
Alessandro Landi, ${ }^{1,}{ }^{*}$ Nicola Marotta, ${ }^{1}$ Cristina Mancarella, ${ }^{1}$ Davide Colistra, ${ }^{1}$ and Roberto Delfini ${ }^{1}$ \\ ${ }^{1}$ Department of Neurology and Psychiatry, Division of Neurosurgery, Sapienza University of Rome, Italy \\ "Corresponding author: Alessandro Landi, Department of Neurology and Psychiatry, Division of Neurosurgery, Sapienza University of Rome, Italy. E-mail: \\ dott.alessandro.landi@gmail.com
}

Received 2015 November 26; Revised 2016 January 21; Accepted 2016 December 06.

\begin{abstract}
Introduction: Minimally invasive percutaneous surgery of the spine is used to treat thoracolumbar junction and lumbar spine fractures by percutaneous fixation. Once fusion has been obtained, it is possible to remove the percutaneous instrumentation after $6-12$ months. We report the case of an obliged removal of the fixation system at 12 months following operation in a patient with a pre-existing compensated and asymptomatic idiopathic scoliosis.

Case Presentation: A 48-year-old patient affected by a compensated asymptomatic idiopathic scoliosis with an L3 type A3 fracture. The patient underwent a percutaneous short fixation L2 - L4. In the following months the patient presented progressive worsening of the low back pain and walking difficulties. The percutaneous fixation system was then removed using the same surgical access. Conclusions: This particular case explains well the importance of biomechanical balance when a spinal fixation should be perform, and demonstrate how an underestimation of this aspect may cause a worsening of symptoms even if the surgical procedure was correctly performed. It is evident that the removal procedure can lead clinical benefit to a patient, in which the fixation system created a decompensation of the curvature of the spine, thus causing biomechanical alterations and generating pain. In these cases, it may be opportune to limit the fracture reduction during the surgical procedure to modify the least possible the pre-existing scoliosis and to increase the patient's comfort after the operation. The biomechanical behaviour of the spine is specific for each patient so only a careful detection of it could lead to an optimal therapeutic result.
\end{abstract}

Keywords: Percutaneous Short Fixation, Thoraco-Lumbar Junction, Burst Fracture, Surgery

\section{Introduction}

Minimally invasive percutaneous surgery of the spine was initially created in the 90's to treat degenerative disc disease using endoscopic means. It subsequently has been used to treat thoracolumbar and lumbar spine fractures. Once fusion has been obtained, it is possible to remove the percutaneous instrumentation after 6 - 12 months. However, often patients refuse this procedure because they do not complain any problems derived from the system. Percutaneous fixation is not free from risks but these are well known and therefore foreseeable. Complications are rare; among these is the biomechanical decompensation of a pre-existing idiopathic scoliosis. We report the case of an obliged removal of the fixation system at 12 months following operation in a patient with a pre-existing compensated and asymptomatic idiopathic scoliosis. This particular case explains well the importance of biomechanical balance when a spinal fixation should be perform, and demonstrate how an underestimation of this aspect may cause a worsening of symptoms even if the surgical procedure was correctly performed.

\section{Case Presentation}

A 48-year-old patient affected by a compensated asymptomatic idiopathic scoliosis (Cobb's angle $23^{\circ}$ - Figure 1 ), reached the emergency department for a 4 metres fall on the sacrum with consequent L3 fracture, definable as an A3 burst fracture according to the Magerl classification. The patient didn't complain any neurological deficit.

According to the algorithm of treatment proposed by our institute [1] the patient underwent a percutaneous short fixation L1 - L3 using Pathfinder Zimmerspine instrumentation implemented with a slight intra-operative distraction and ligamentotaxis to reduce the fracture.

A post-operatory X-ray showed a partial realignment of the pre-existing scoliosis (Cobbs' angle $15^{\circ}$ - Figure 2 ), reduction of the fracture and the correct positioning of the system. Moreover he underwent TC scan that showed no radiological evidence of neurological compression, breakage or dislocation of the system.

In the following months the patient, at 1, 3 and 8 months, underwent clinical and radiological follow-up through radiological and clinical examination. The X- ray controls (1, 3 and 8 months) showed complete ossification 


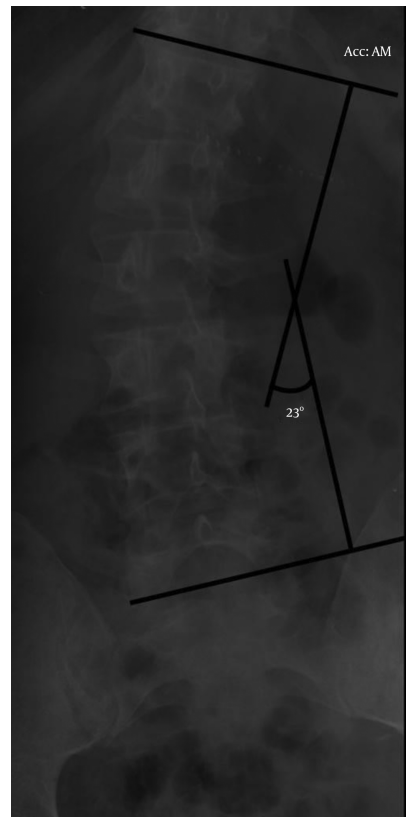

Figure 1. X-Ray Showing the Compensated Asymptomatic Idiopathic Scoliosis (Cobb's Angle $23^{\circ}$ )

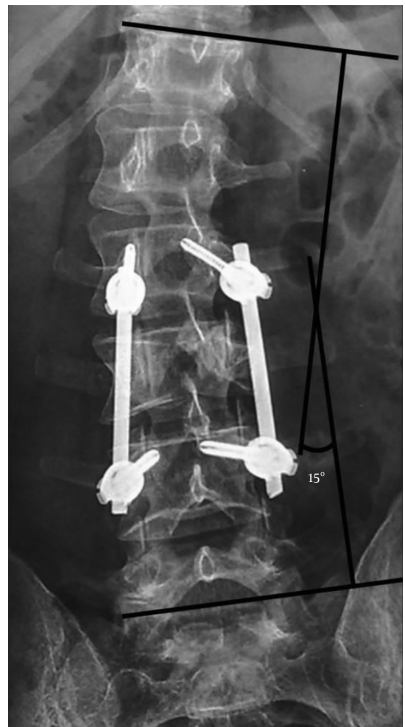

Figure 2. Post Operatory X-Ray Showing a Partial Realignment of the Pre-Existing Scoliosis (Cobbs' Angle $15^{\circ}$ )

of the fracture even though the patient still referred constant back pain. Clinically he presented progressive worsening of the low back pain and walking difficulties evaluated by the VAS score and ODI scale. Clinical and radiological findings together with the patient's wish made us decide to remove the percutaneous fixation system using the same surgical access. The patient was discharged after 3 days.

One month after the procedure the patient reported back pain relief (decrease of the VAS score and ODI value) and an X-ray evidenced the return to the pre-exiting scoliosis curvature (Cobb's angle $21^{\circ}$ - Figure 3 ) confirmed on an $\mathrm{X}$-ray made after 6 months. At six months were symptoms free.

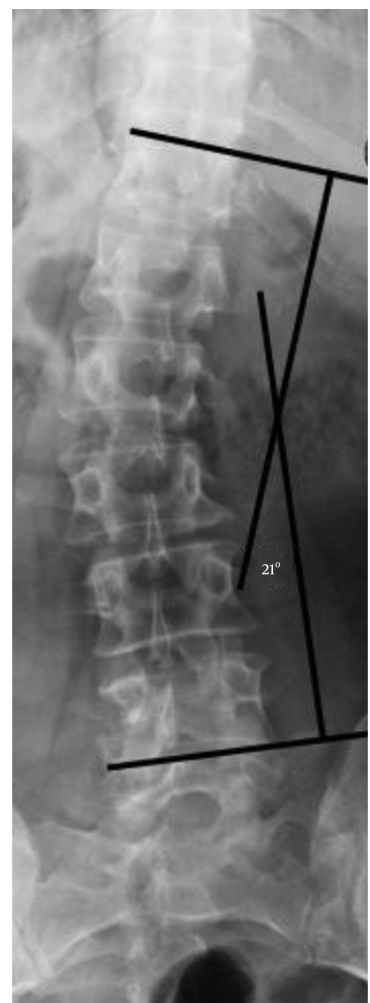

Figure 3. The X-Ray Performed After the Removal of the System Evidenced the Return to the Original Scoliosis Curvature (Cobb's Angle $21^{\circ}$ )

\section{Discussion}

Percutaneous stabilization is performed in case of thoracolumbar junction or lumbar trauma. In the case reported the patient presented an A3 type fracture according to the Magerl classification, and it was not necessary to perform a postero-lateral fusion because of the integrity of the posterior tension band [2]. For this type of fracture the gold standard treatment is the use of a surgical or thesis [3], even if the functional improvement takes more time and the management of pain and mobility is less satisfying $[4,5]$. 
In case of percutaneous stabilization, removal of the system is indicated once fusion has taken place with the goal to re-establish the correct range of movement of the area involved. However, some patients refuse this procedure because of the resolution of the symptoms. It is possible that in patients with lumbar column dysmorphia, the stabilization may alter the biomechanics of the column causing pain.

In case of mild scoliosis, as in our patient, the reduction and distraction of the fracture and the realignment of the segment can correct the scoliosis. This procedure relieves the pressure on the joint but overcharges the contralateral causing excessive stress on this one during abnormal solicitation. Furthermore the short fixation leads to the block of two motor units: L2 - L3 and L3 - L4. Normally, the range of movement of the immobilized segments can cause increased movements of the adjacent superior and inferior segment [6] as mechanism of compensation. In the case of our patient the realignment of the scoliosis caused an axial overload that caused an increased range of motion of the segment. This process brings to a greater stress on the articular joint, on the disc, on the adjacent segment and on the posterior ligamentous structures, causing edema of the interspinous ligament. In addition, such overload can lead to capsule-ligament looseness, increase of the intra-articular fluid and micro-instability. All these events can represent the beginning of a degenerative cascade [7].

In some cases the correction of the scoliosis leads to new compensation, the spine assumes a correct posture and the axial, rotational and dynamic load are equally distributed on all the structures responsible for the motion control, which brings to the resolution of the symptoms.

In other cases, when conservative treatment fails, the only way to re-establish the biomechanical balance, to reduce the symptoms and to prevent the onset of plurimetameric degeneration, is to re-establish the preexisting physiological curvature and so re-set the scoliosis compensation. In these cases, the removal of the system after consolidation of the fracture can lead to resolution of the symptoms, as in the case described. Furthermore, the removal procedure is extremely simple and has few risks through the same surgical access.

Clearly this is only a report of a personal experience. To validate this theory a well-designed control trial to explain its effectiveness should be made. With a longer follow- up for pain relief to confirm its benefit.

\subsection{Conclusion}

The clinical advantage of the removal of percutaneous fixation system in traumatic pathology, as opposed to its maintenance in the patient, is still not clear. From a biomechanical aspect it is important to re-set the physiological motility of the motor unit involved. The importance of biomechanical balance when a spinal fixation should be perform, must always be taken in to consideration because an underestimation of this aspect may cause a worsening of symptoms even if the surgical procedure was correctly performed. It is evident however that this removal procedure can lead clinical benefit to a patient in which the fixation system created a decompensation of the curvature of the spine, thus causing biomechanical alterations and generating pain. In these cases, it may be opportune to limit the fracture reduction during the operation to modify the least possible the pre-existing scoliosis and to increase the patient's comfort after the operation. The biomechanical behaviour of the spine is specific for each patient so only a careful detection of it could lead to an optimal therapeutic result.

\section{Footnote}

Authors' Contribution: Alessandro Landi and Nicola Marotta perform surgery, Davide Colistra and Cristina Mancarella wrote the article, Alessandro Landi and Roberto Delfini reviewed the article and the typesetting, AL and CM performed the study design

\section{References}

1. Marotta N, Landi A, Delfini R. Algorhythm for Use of Percutaneous Short Fixation of Fractures Involving the Thoracolumbar Junction and Lumbar Spine. Int J Clin Med. 2013;4(7) doi:10.4236/ijcm.2013.47a1003.

2. Vaccaro AR, Oner C, Kepler CK. AOSpine thoracolumbar spine injury classification system: Fracture description, neurological status and key modifiers. Philadelphia: Spine; 2013.

3. Ghobrial GM, Jallo J. Thoracolumbar spine trauma: review of the evidence. J Neurosurg Sci. 2013;57(2):115-22. [PubMed: 23676860].

4. Bailey CS, Dvorak MF, Thomas KC, Boyd MC, Paquett S, Kwon BK, et al. Comparison of thoracolumbosacral orthosis and no orthosis for the treatment of thoracolumbar burst fractures: interim analysis of a multicenter randomized clinical equivalence trial. J Neurosurg Spine. 2009;11(3):295-303. doi: 10.3171/2009.3.SPINE08312. [PubMed: 19769510].

5. Dai LY, Jiang LS, Jiang SD. Conservative treatment of thoracolumbar burst fractures: a long-term follow-up results with special reference to the load sharing classification. Spine (Phila Pa 1976). 2008;33(23):253644. doi: 10.1097/BRS.0b013e3181851bc2. [PubMed: 18978595].

6. Ha KY, Son JM, Im JH, Oh IS. Risk factors for adjacent segment degeneration after surgical correction of degenerative lumbar scoliosis. Indian J Orthop. 2013;47(4):346-51. doi: 10.4103/0019-5413.114912. [PubMed: 23960277].

7. Blondel B, Fuentes S, Pech-Gourg G, Adetchessi T, Tropiano P, Dufour H. Percutaneous management of thoracolumbar burst fractures: Evolution of techniques and strategy. Orthop Traumatol Surg Res. 2011;97(5):527-32. doi:10.1016/j.otsr.2011.03.020. [PubMed: 21763230]. 